\title{
Mayana Katz, Ketih Okamoto: Stem cells in modeling human genetic diseases
}

\author{
Christian Schnell ${ }^{1}$
}

Published online: 13 November 2015

(C) Springer-Verlag Berlin Heidelberg 2015

The book "Stem Cells in Modeling Human Genetic Diseases" was published in 2015 and edited by Mayana Katz and Keith Okamoto both from the University of Sao Paulo in Brazil. They brought together more than 20 experts on the recent developments around stem cells and how they can help us to understand human genetic diseases. The book covers a range of topics from cardiomyopathies, neurological diseases and also cancer stem cells and aims to provide an overview of the use of stem cells in these fields.

The diseases covered in detail are fragile X syndrome, familial dilated cardiomyopathy, amyotrophic lateral sclerosis, spinocerebellar ataxia, vascular disease, cardiomyopathies, cancer stem cells and chemoresistance and autism spectrum disorders. On the back of the book also diseases such as Huntington's disease, age-related macular degeneration and schizophrenia are mentioned as being explored in detail in the book. However, most of them are not mentioned in the index at all and are only mentioned secondarily or in passing - which is a bit misleading. Readers with a particular interest in those diseases are probably not the target audience of this book.

There are certainly more genetic diseases for which stem cells are used for modeling than are mentioned in the book. Still, the range of topics covered in the book is quite broad and diverse, so the reader will get a good overview about the current state of the use of iPS technology in the fields mentioned in the book. Each chapter starts with an introduction to the disease and explains the underlying genetic causes. Most chapters also contain a couple of paragraphs dealing with the generation of iPS in general and the protocols used for the generation of the cell type of interest from the iPS. But the book is not supposed to be an introduction to iPS technology in general. Most of the authors compare the use of iPS for modeling the disease to the available animal models. Furthermore, in many chapters the authors also mention the drawbacks and challenges of using iPS, being aware that the way for potential clinical interventions based on iPS is still a long way to go in the vast majority of diseases mentioned in the book.

The target audience of this book are students, researchers and clinicians interested in the recent developments in the field. The book is well written and, thanks to the good introductory section in each of the chapters, even non-experts in the field will be able to follow. The figures in the book are well-designed and the quality of the print is also good, including the immunohistochemical images that are shown in some of the chapters. The book gives a good overview about the current state of research in these fields and can therefore be recommended for interested readers.

Christian Schnell

SchnellC@ cardiff.ac.uk

1 Cardiff School of Biosciences, Cardiff University,

The Sir Martin Evans Building, Museum Avenue,

Cardiff CF10 3AX, UK 